\title{
Combustion and microexplosion of collision-merged methanol/alkane droplets
}

\author{
C.H. Wang ${ }^{\mathrm{a}, *}$, S.Y. Fu ${ }^{\mathrm{a}}$, L.J. Kung ${ }^{\mathrm{a}}$, C.K. Law ${ }^{\mathrm{b}}$ \\ ${ }^{a}$ Department of Mechanical Engineering, National Taiwan University, Taipei, Taiwan, ROC \\ ${ }^{\mathrm{b}}$ Department of Mechanical and Aerospace Engineering, Princeton University, Princeton, NJ 08544, USA
}

\begin{abstract}
The combustion characteristics of freely falling droplets, individually generated by the merging of colliding methanol and alkane droplets, were investigated and compared with those for pure methanol and alkanes. The merging of the nominally immiscible methanol and alkanes was manifested in an apparently adhesive, but unmixed, manner in all test conditions. An air bubble was found to be trapped at the colliding interfaces where they were "adhered," with the trapping favored for head-on or near head-on collision orientations. The trapped air bubble ostensibly induced heterogeneous nucleation of the methanol, being facilitated by the relatively low limit of superheat of methanol. Consequently, the droplet exploded almost immediately upon ignition, leading to an extremely short overall lifetime. For collision orientations that were more off-centered, bubble trapping and thereby heterogeneous nucleation were not favored. However, delayed, albeit strong, microexplosion occurred through homogeneous nucleation of methanol at the contacting interface. The global burning rate was therefore again augmented. In general, microexplosion was facilitated for high-boiling-point alkanes such as hexadecane and tetradecane. The co-vaporization of methanol and alkane from their respective hemispherical segments constituting the adhered droplet also led to flame colors that were more bluish than yellowish, indicating the reduction of soot from alkane burning in the presence of methanol vapor. In light of the difficulty of forming stable methanol/oil emulsions, the potential of separate injection of oil and methanol in opposed jet arrangement, in direct-injection engines to facilitate collision, is suggested.

(C) 2004 The Combustion Institute. Published by Elsevier Inc. All rights reserved.
\end{abstract}

Keywords: Droplet combustion; Methanol combustion; Droplet collision

\section{Introduction}

It has been quite extensively demonstrated that the utilization of water/oil emulsions in internal combustion engines can significantly reduce the various deleterious heterogeneous combustion ef-

\footnotetext{
* Corresponding author. Fax: +886 0223631755.

E-mail address: chinghua@ccms.ntu.edu.tw (C.H. Wang).
}

fects associated with spray combustion [1-7]. Such beneficial effects have been attributed to several factors. Foremost among them is the potential occurrence of droplet microexplosion due to the superheating [8-12], and thereby internal gasification, of the embedded water micro-droplets dispersed within a high-boiling-point oil such as diesel or hexadecane. This secondary atomization event reduces the extent of heterogeneity and promotes micro-scale mixing. Furthermore, gasification of water from the emulsion droplet reduces 
the flame temperature and increases the concentrations of the $\mathrm{OH}$ radical in the fuel-rich region of the flame, which tend to reduce the formation of $\mathrm{NO}_{x}$ and soot precursors, respectively. These effects are particularly strong when the droplets burn either individually or in groups because modifications of the flame temperature and $\mathrm{OH}$ concentration take place in the droplet vicinity. Fundamental studies have also shown that the intensity of microexplosion is much stronger for emulsions than for miscible mixtures, hence implying the desirability that the additive be implemented as an emulsion instead of a miscible fuel blend, provided emulsion stability is assured.

Since water does not have any heating value, the energy density of water/oil emulsions is reduced from those of the neat fuels. This factor could outweigh the beneficial effects mentioned above when fuel weight and travel range are important considerations. It has therefore been suggested that methanol, which is also immiscible with oil but is otherwise a fuel, be used in the form of methanol/oil emulsions. Indeed, it appears that the various benefits associated with water/oil emulsion are largely retained with methanol/oil emulsions. Specifically, since the limit of superheat of methanol is between 440 and $460 \mathrm{~K}$ [13], which is substantially lower than that of water $(560-580 \mathrm{~K})$, the potential for microexplosion could actually be enhanced, especially for oils that have lower boiling points than those of hexadecane and diesel. This could therefore extend the range of fuel volatility that can be benefited with the emulsification. Furthermore, the flame temperature is also expected to be reduced from that of the neat fuel, though to a smaller extent than water, because of the smaller heat of combustion of methanol as compared to those of conventional oils. Kinetically, since $\mathrm{OH}$ radicals are also generated in substantial amounts in reactions involving methanol, oxidation of the soot precursors could still be facilitated. Finally, since methanol can be formulated through, say, coal gasification and biomass conversion, it constitutes a fuel source that is not petroleum based and as such is considered to be a valuable alternate fuel.

Recognizing such potential benefits by using methanol/oil emulsions, attempts at forming stable emulsions of oil with methanol were however found to be futile, even with massive amount of emulsifier addition up to $30 \%$ [10]. Phase separation occurred readily and rapidly upon emulsification. Interest and hence investigation on methanol/oil emulsion combustion have therefore been dormant since the late 1970s.

Recent studies on the seemingly unrelated problem of droplet-droplet collision have however led us to a renewed interest in the potential of methanol/oil droplet combustion. Particularly, it was found [14] that when two droplets of dissimilar but miscible liquids collide, the collision could result in merging, bouncing, merging again, and merging followed by separation as the collision inertia in terms of the droplet Weber number increases. These results are similar to the collision between droplets of the same liquid [15-17]. It was also found that the merged droplets could subsequently undergo microexplosion, which was facilitated by the trapped air bubble between the colliding interfaces, with the trapped bubble serving as the heterogeneous nucleation site.

Results on the collision between a droplet of high-boiling-point hydrocarbon, say hexadecane, and a water droplet, however, exhibited quite distinctively different behavior. It was observed [18] that, upon contact, either these two nominally immiscible liquid droplets were united in an adhesive manner (see Fig. 1), or the water droplet would penetrate into the hexadecane interior and subsequently become enclosed, provided the water volume fraction is less than 0.54 so that it can be completely enveloped. The insertive merging mode prevails for burning situations. Microexplosion of the merged mass occurs upon complete vaporization of the enveloping hexadecane such that the embedded superheated water droplet flash vaporizes.

Recognizing the immiscible nature between methanol and oil, the above results on the combustion and microexplosion responses of collision-generated water-oil droplets suggest that a fresh consideration of the potential of methanol addition in engine combustion is warranted. That is, since stable emulsions of methanol and oil cannot be formed, methanol addition can be implemented through separate injection.
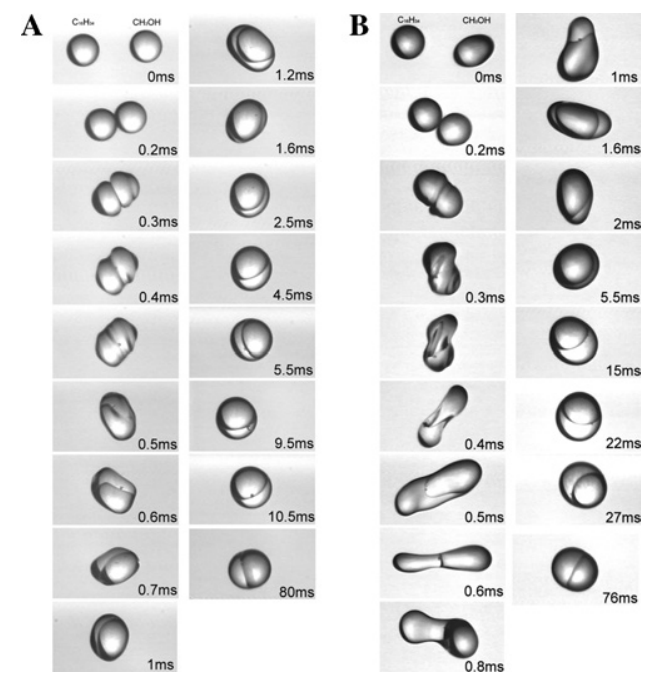

Fig. 1. Photographic images of the collision sequences between methanol and hexadecane droplets of nearly equal sizes, with (A) almost head-on and (B) off-centered collision. 
Consequently, if the injection process can be configured such that collision between methanol and oil droplets is enhanced, say through an opposed jet arrangement, and if their collision responses are similar to those of water and oil, then it is conceivable that microexplosion can again be induced, leading to reduction of the various deleterious heterogeneous effects. Furthermore, if the collision between the methanol and oil droplets results in the adhesive mode, which is indeed the case as we shall show later, then the potential to reduce soot and $\mathrm{NO}_{x}$ formation from droplet or group flames is also realized because both methanol and oil will vaporize together.

In view of the above considerations, the primary objective of the present investigation was to explore and to characterize the collision, combustion, and potential microexplosion processes of collision-generated methanol/alkane droplets. We shall show in due course that strong microexplosion of such droplets is indeed possible, thereby providing motivation for further fundamental and practical studies for its potential utility in engines. We note in passing that the possible occurrence of droplet collision in engines was suggested in $[19]$.

In the following, we shall first present the experimental specifications, which are followed by presentation of the experimental results on the collision outcome, the flame and microexplosion characteristics, and the combustion characteristics including the ignition delay, the extinction states, and the burning rates.

\section{Experimental apparatus and procedure}

The experimental apparatus and procedure were described in $[14,20]$, and hence will be only briefly discussed here. Specifically, two independently controlled droplet streams with the same droplet generation rate were produced using a modified ink-jet printing technique, and were made to collide in the desired manner by manipulating the phase difference and/or the relative positions of the two generators. The collided droplets then fell through a small vertical connecting channel into a burner-generated, high-temperature, oxidizing environment in which the droplets ignited and burned. The droplets entered the combustion chamber with a velocity of $\sim 70 \mathrm{~cm} / \mathrm{s}$, while the environment had a maximum temperature of $\sim 1300 \mathrm{~K}$ with an oxygen concentration of 23.5 vol.\%. The maximum Reynolds number of the droplet is $\mathrm{O}(1)$.

A strobe light synchronized with the droplet generators, with variable phase lag, was used to freeze the moving droplets at various stages of their lifetime. Enlarged droplet images were recorded by a CCD camera with a Baush \& Lomb Mono-zoom-7 long focus microscopic lens. The uncertainty in the determination of the droplet size was estimated to be about $2 \%$, mainly from reading the boundary of the droplet image.

The droplet size was controlled by altering the size of the glass nozzle. In the experiments, the volume fraction of methanol was controlled to be $\sim 0.25,0.5,0.75$, and 0.82 such that the final merged volume was equivalent to that of a droplet with a diameter of $\sim 0.320 \mathrm{~mm}$. Data for pure methanol and alkane droplets with the same diameter were also determined for comparison. Technical grade hexadecane, tetradecane, dodecane, decane, and methanol with purity greater than $99 \%$ were used as the test fuels.

In the present study, the droplet burning characteristics such as the flame appearance, ignition delay, burning times and rates, and also the state of microexplosion were investigated. The ignition delay was defined as the time interval from the instant at which the droplet was first exposed to the high-temperature environment to the instant when a visible flame was observed. The droplet burning process was assessed through visual observation of the flame streak and microphotography of the droplet images.

The experimental resolution of the time scale of the electronic device was $\pm 0.04 \mathrm{~ms}$, and the uncertainty in defining the ignition point was estimated to be $\pm 1 \mathrm{~ms}$. The droplet generation frequency was about $10 \mathrm{~Hz}$, such that the droplets were separated by over several hundreds of diameters to minimize the effect of droplet interaction.

It is to be noted that because of the repeatability and stability of the droplet streams, each datum point in the following figures actually represents thousands of test droplets. Since the collision-generated droplets were not in perfect spherical shape, particularly at low temperatures, the equivalent droplet size, based on the total volume of the colliding methanol and alkane droplets, was adopted.

\section{Results and discussion}

\subsection{Collision outcomes}

Figures $1 \mathrm{~A}$ and $\mathrm{B}$ show the photographic images of the collision sequences between methanol and hexadecane droplets of nearly equal sizes, 0.258 vs. $0.256 \mathrm{~mm}$, with almost head-on and offcenter collisions, respectively. The collision images are similar for other alkanes such as tetradecane, dodecane, and decane. For those collision events that did not result in bouncing, the two colliding droplets simply stayed in contact, in the adhesive mode as observed for water-alkane droplets [18], with a distinct contact surface between the two immiscible components. However, unlike the collision between water and alkane droplets, the insertive mode of merging was not observed. This 

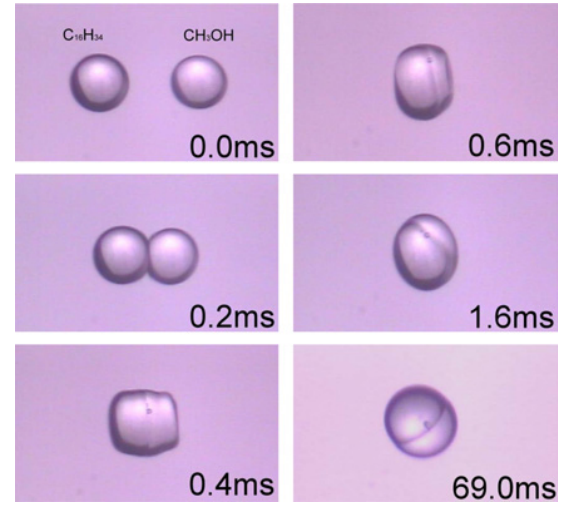

Fig. 2. Enlarged photographs showing trapped air bubbles.

can be explained by the difference between their surface tensions. Particularly, the surface tension values for hexadecane, water, and methanol at room temperature are $0.0275,0.0729$, and $0.0226 \mathrm{~N} / \mathrm{m}$, respectively. Since the surface tensions for hexadecane and methanol are of comparable magnitude, the colliding droplets tend to spread out upon contact. The same observation holds for other alkanes because they have similar values of surface tension. However, water has a substantially larger surface tension than hexadecane. Consequently, it tends to retain its contracted shape upon droplet-droplet contact, and penetrates into the interior of the hexadecane droplet if there is sufficient hexadecane mass to spread around the water element.

The recorded images also show the presence of a "black" spot attached to the contacting surface of the coalescent droplets, as clearly shown in Fig. 2. This black spot is attributed to be the air bubble trapped between the colliding surfaces upon merging, as it is well established that bubble trapping is an integral part of the droplet collision and coalescence process. Extensive exploration demonstrated that while the trapping always occurred for head-on and near head-on collisions, the presence of the air bubble gradually diminished as the collision becomes more off-center. This trapped air bubble plays an essential role in initiating internal gasification, and hence droplet microexplosion, by serving as a heterogeneous nucleation site of the superheated liquid element interior of the merged mass, as we shall show later.

\subsection{Flame characteristics}

Once the merged droplet was ignited, a steady flame streak was observed. For all the pure alkane droplets, the flames were blue for a short distance from ignition. It then became white-yellow inside and blue outside for a large portion of the burning trajectory, and finally turned to blue again until the very end of the flame. For methanol droplets, however, the flame was almost invisible and extinguished shortly before the droplet was completely gasified. These are shown in Figs. 3A and D, respectively.

For the methanol/alkane droplets, the flame behaviors can be distinguished into the following three categories: (1) early explosion mode (Fig. 3B), which occurred almost immediately after the collision-generated droplet was ignited and was accompanied by several small branched flames; (2) late explosion mode (Fig. 3C), in which a regular flame streak existed for a while and then terminated with a flame ball; and (3) non-explosive mode, in which the droplet burned out either quietly or in the form of a tiny yellow sparkling flame whose intensity was much weaker than that of the late explosion mode.

The critical factor that determines whether the merged droplet underwent early or late explosion is the extent of off-centeredness upon collision. Specifically, early explosion invariably occurred for head-on or near head-on collisions, while the explosion was delayed as the collision became more off-centered.

The intensity of explosion depended on the relative sizes of the two droplets and the specific alkane in terms of its boiling point. Table 1 summarizes the dependence of the nature of the explosion on these two factors. The reasons for the observed behavior will become clear as we discuss the mechanisms of explosion in the next section.

Finally, since methanol and alkane each constitutes a "hemispherical" sector of the merged mass, these two components can each vaporize
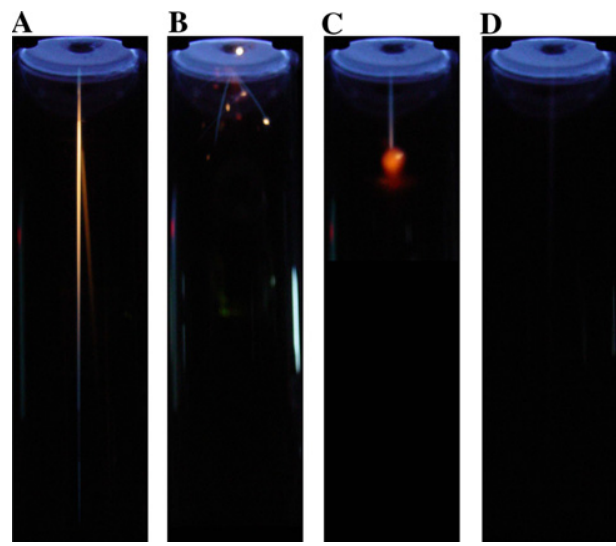

Fig. 3. Flame images of collision-merged methanol/ hexadecane droplet streams: (A) non-explosive and sooty flame of pure hexadecane; (B) early explosion mode of $50 / 50 \%$ methanol/hexadecane; (C) late explosion mode of $50 / 50 \%$ methanol/hexadecane; and (D) non-explosive, non-sooty flame of pure methanol. 
Table 1

Summary of explosion intensity for different alkanes and methanol fraction

\begin{tabular}{llll}
\hline & $\sim 25 \%$ Methanol & $\sim 50 \%$ Methanol & $\sim 75 \%$ Methanol \\
\hline Hexadecane & Early explosion or & Early explosion or strong & Early explosion or weak \\
& strong late explosion & late explosion & Early explosion \\
Tetradecane & Early explosion or & Early explosion or weak & late explosion \\
& strong late explosion & late explosion & Early explosion or weak \\
Dodecane & No early explosion, & Early explosion or weak & late explosion \\
& very weak late explosion & late explosion & No early explosion, very weak \\
Decane & No early explosion, & No early explosion, very & late explosion \\
& very weak late explosion & weak late explosion & \\
\hline
\end{tabular}

freely, with the vaporization rate varying over the droplet surface, being dependent on their individual boiling points and the heat transfer rate between them. Consequently, the burning rate and flame intensity in terms of its color are also not uniform over the flame surface, being intermediate between those of methanol and alkane, as anticipated earlier.

\subsection{Microexplosion}

The observations that an air bubble is trapped for near head-on collisions, which tend to undergo early explosion, and that it is not trapped for sufficiently off-centered collisions, which tend to undergo late explosion, provide collaborating evidence on the nature of the respective explosions. Specifically, it is reasonable to suggest that the early and late explosions are, respectively, heterogeneous and homogeneous in nature, with the trapped air bubble serving as heterogeneous nucleation site for the former. Thus, if an air bubble is trapped upon merging, then heterogeneous nucleation can be readily induced as the droplet is ignited, and hence heated, leading to early explosion. The extent of the superheat accumulated at nucleation, and hence the intensity of explosion, increases with increasing boiling point of the alkane. Consequently, hexadecane and tetradecane exhibit strong early explosions. As for dodecane, early explosion was observed for $0.5,0.75$, and 0.82 volume fractions of methanol, indicating that the contact surface temperature could reach a point to induce sudden vaporization of methanol. For decane, early explosion was not observed for all methanol contents. Thus, the possible trapping of an air bubble at the contact surface and the temperature distribution associated with the relative contents of methanol to alkanes, as well as the specific alkane, determine the appearance of early explosion.

For sufficiently off-centered collision, since trapping of the air bubble was not favored, heterogeneous nucleation and thereby early explosion were not observed. A prolonged period of burning therefore ensued. At a certain stage, the methanol interface could be heated to the limit of superheat, upon which it would undergo homogeneous nucleation and again induce micro-explosion. Similar to the heterogeneous case, the controlling factor here is the temperature distribution within the merged droplet. However, a higher interfacial temperature is needed to induce homogeneous nucleation. Assuming that the methanol and alkane segments can each attain a maximum temperature close to its boiling point at their respective evaporating surfaces, the interior temperature distribution would then vary from $338 \mathrm{~K}$ on the methanol side to $447,489,527$, and $560 \mathrm{~K}$ on the alkane side for decane, dodecane, tetradecane, and hexadecane, respectively. Since the limit of superheat for methanol is 440 $460 \mathrm{~K}$, it is reasonable to expect that late explosion is unlikely for decane and dodecane, and is possible for tetradecane and hexadecane. Indeed, strong late explosion was experimentally observed for hexadecane with 0.25 and 0.5 volume fractions, and for tetradecane with 0.25 volume fraction. Only weak explosions characterized by tiny sparkling flame balls were observed for other concentrations of hexadecane and tetradecane, and for all concentrations of dodecane and decane. Such weak explosions are likely caused by the concentrated impurity or the condensation of water vapor [21] or reaction intermediates [22]. The droplet size at the point of this weak explosion is less than $\sim 0.05 \mathrm{~mm}$, and the corresponding volume is less than $0.4 \%$ of the initial volume.

Figure 4 shows the zoomed droplet explosion images for the early and late explosions for meth-
$\mathbf{A}$

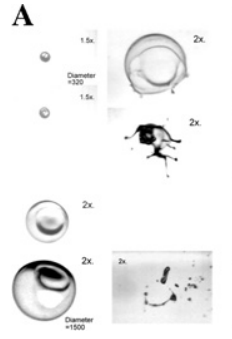

B

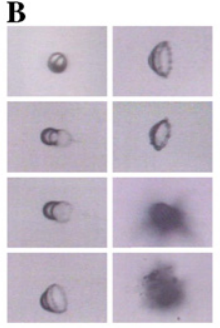

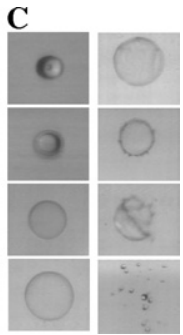

Fig. 4. Zoomed images of: (A) early explosion of methanol/hexadecane droplet; (B) late explosion of methanol/hexadecane droplet; and $(C)$ explosion of the miscible heptane/hexadecane droplet. 
anol/hexadecane droplets, and explosion for a droplet generated by the collision of miscible heptane/hexadecane droplets. It is seen that the explosion starts at the interface for the methanol/alkane droplet, such that the vaporized methanol is partly enclosed by the crown and the thin layer of liquid methanol, but is away from the center for the miscible heptane/hexadecane droplet.

\subsection{Ignition delays}

Figure 5 plots the ignition delays of methanol/ alkane droplets for various volume fractions. All data were obtained for an initial droplet diameter of $0.320 \mathrm{~mm}$. Results show that the ignition delay for pure methanol is shorter than those of the pure alkanes. Furthermore, a maximum in the methanol volume fraction exists for methanol/alkane droplets, which becomes more prominent as the alkane becomes more volatile. This nonmonotonic result can be explained by recognizing the fact that methanol has a lower boiling point than the alkanes, and hence a shorter thermal ignition delay [23] is needed to heat up the droplet, while the alkanes have shorter chemical ignition delays to initiate gas-phase reaction. For the latter factor, we note that the auto-ignition temperatures for hexadecane and dodecane have been reported [24] to be in the range of $480-500 \mathrm{~K}$, while that of methanol is $660-850 \mathrm{~K}$. While substantial variations exist for the methanol values, it is nevertheless obvious that the auto-ignition temperature for hexadecane is lower than that of methanol.

\subsection{Extinction}

As expected, all pure alkane droplets basically burned to completion. For the methanol/alkane droplets, burning could be terminated by early

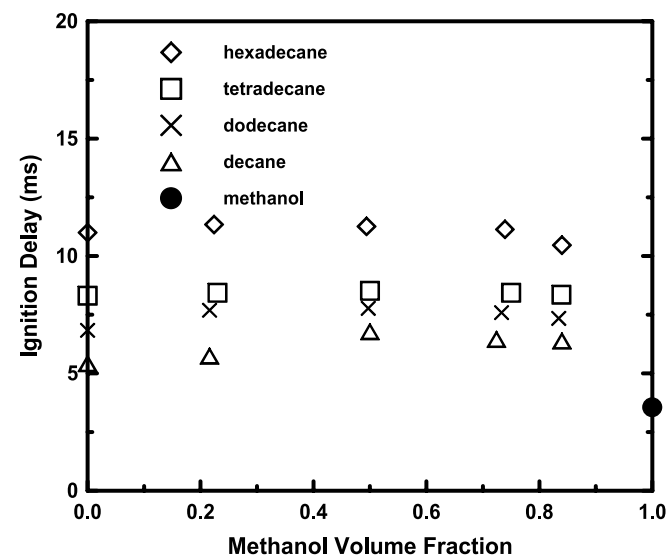

Fig. 5. Ignition delays of methanol/alkane droplets for various volume fractions $\left(D_{\mathrm{o}}=0.320 \mathrm{~mm}\right)$. explosion, late explosion, complete droplet consumption, or almost complete droplet consumption accompanied by a weak explosion. For pure methanol droplets, however, extinction occurred with an unburned droplet of substantial size. For an initial droplet size of $0.320 \mathrm{~mm}$, the extinction droplet size was $\sim 0.106 \mathrm{~mm}$. This extinction was caused by the accumulation of water through its condensation at the droplet surface and subsequently dissolution into the droplet interior, as noted previously [21].

\subsection{Burning times and rates}

Defining the burning time as the period between the appearance and disappearance of a flame, Fig. 6 shows the burning times of droplets with various methanol volume fractions. The exceptionally small values for methanol/hexadecane are consequence of the occurrence of strong (late) explosions. We also note that the burning times of the various pure alkanes are quite close, which is reasonable. The lower value for pure methanol as compared to the pure alkanes is due to the occurrence of water-induced extinction, recognizing nevertheless that the droplet is not completely gasified at the instant of extinction.

In determining the burning times for the various fuels and burning situations, we have not considered the cases in which early explosion occurred. For such situations, the burning time is extremely short, and there is great uncertainty in the accuracy and repeatability in the droplet size measurement. On the other hand, an accurate knowledge of this quantity is not needed because for all practical purposes the droplet can be considered to be gasified instantly.

Because of the complexity of the problem, a detailed, $\mathrm{d}^{2}$-law type analysis cannot be readily performed to yield meaningful burning rate con-

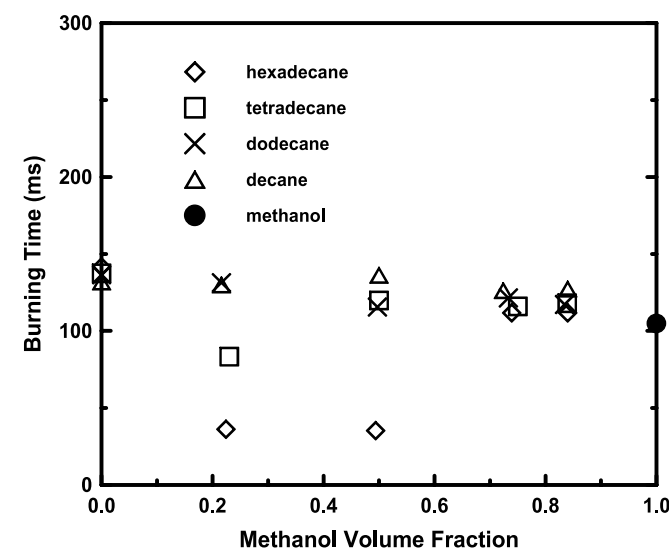

Fig. 6. Burning times of methanol/alkane droplets for various volume fractions $\left(D_{\mathrm{o}}=0.320 \mathrm{~mm}\right)$. 


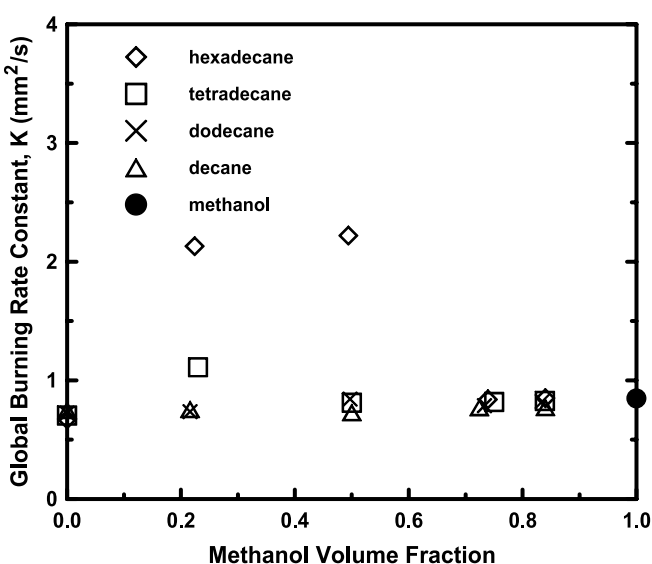

Fig. 7. Global burning rates of methanol/alkane droplets for various volume fractions $\left(D_{\mathrm{o}}=0.320 \mathrm{~mm}\right)$.

stants. Instead, the effects of all processes can be grouped into a global burning rate constant $K$, defined as

$K=D_{\mathrm{o}}^{2} / \Delta t$

where $D_{\mathrm{o}}$ is the initial diameter of the merged droplet, and $\Delta t$ is the sum of the ignition delay time and the burning time. Figure 7 plots the burning rates for the variations situations of Fig. 6 . The very large values for methanol/hexadecane droplets, due to the occurrence of strong microexplosion, are to be noted.

\section{Concluding remarks}

Results and understanding gained from the present study can be summarized as follows:

1. Droplets of methanol and oil, alkane in the present investigation, can merge but not mix upon collision. Merging is in an adhesive mode, with the resulting mass consisting of two hemispherical-like portions "adhered" at their interface.

2. An air bubble can be trapped at the interface of the colliding droplets when they merged. Trapping is favored head-on or near head-on collisions.

3. Head-on or near head-on collisions can result in early microexplosion, which occurs almost immediately after ignition, and is believed to be caused by the heterogeneous nucleation of the trapped air bubble at the methanol/alkane interface.

4. Sufficiently off-centered collisions can result in late microexplosion of strong intensity, and is believed to be caused by the homogeneous nucleation of methanol at the methanol/alkane interface.
5. Nucleation and hence microexplosion are favored for high-boiling-point alkanes, especially hexadecane and tetradecane.

6. The ignition delays of alkane droplets are all longer than that of methanol due to the need for droplet heating of these high-boiling-point fuels. However, the ignition delays of methanol/alkane droplets are even longer due to the combined effects of thermal ignition delay caused by the alkanes and chemical ignition delay caused by methanol.

7. Methanol droplet burning is non-sooty, but is incomplete due to early extinction. Alkane droplet burning is basically complete, but is sooty. The burning of the merged methanol/alkane droplet is quite non-sooty and is complete, with or without microexplosion.

8. The occurrence of early microexplosion leads to extremely short droplet lifetime, and hence rapid rate of heat release. The occurrence of strong late explosion also doubles or even triples the global burning rate.

9. Based on the results of tetradecane and hexadecane, the potential of rapid burning rates and soot reduction through separate methanol injection, configured to enhance droplet collision, merits further study for applications in direct-injection engines.

\section{Acknowledgments}

The work at the National Taiwan University was supported by the National Science Foundation of Taiwan, Republic of China, and that at Princeton University was supported by the US Air Force Office of Scientific Research under the technical monitoring of Dr. Mitat Birkan.

\section{References}

[1] I. Cornet, W.E. Nero, Ind. Eng. Chem. 47 (1955) 2133-2141.

[2] E. Valdmanis, D.E. Wulfhorst, SAE 700736 (1970).

[3] D.W. Turner, C.W. Siegmund, in: Presented at the Winter Symposium of the IEC Division of the American Chemical Society, 1973.

[4] G. Greeves, I.M. Khan, G. Onion, Proc. Combust. Inst. 16 (1976) 321-336.

[5] E.C. Owens, B.R. Wright, AFLRL 83, 1976.

[6] T. Murayama, SAE 780224 (1978).

[7] C.H. Wang, L.H. Ni, Chin. J. Mech. 12 (4) (1996) 465-474.

[8] C.T. Avedisian, R.P. Andres, J. Colloid Interface Sci. 64 (3) (1978) 438-452.

[9] J.C. Lasheras, A.C. Fernandez-Pello, F.L. Dryer, Combust. Sci. Technol. 21 (1979) 1-14.

[10] J.C. Lasheras, A.C. Fernandez-Pello, F.L. Dryer, Proc. Combust. Inst. 18 (1981) 293-305. 
[11] C.H. Wang, C.K. Law, Combust. Flame 59 (1985) $53-62$.

[12] C.H. Wang, J.T. Chen, Int. Comm. Heat Mass Transfer 23 (1996) 823-834.

[13] M. Blander, J.L. Katz, AICHE J. 21 (1975) 833-848.

[14] C.H. Wang, W.G. Hung, S.Y. Fu, W.C. Huang, C.K. Law, Combust. Flame 134 (2003) 289-300.

[15] N. Ashgriz, J.Y. Poo, J. Fluid Mech. 221 (1990) 183-204.

[16] Y.J. Jiang, A. Umemura, C.K. Law, J. Fluid Mech. 234 (1992) 171-190.

[17] J. Qian, C.K. Law, J. Fluid Mech. 331 (1997) 59-80.

\section{Comment}

Mary Julia (Judy) Wornat, Louisiana State University, USA. This is a very interesting and instructive piece of work. You have shown that two droplets of immiscible liquids will, upon collision, adhere to one another. Down to what droplet size would you expect this behavior to be displayed? To what extent, if any, is the phenomenon dependent on the physical properties of the two immiscible liquids chosen?

Kalyan Annamalai, Texas A\&M, USA. Typically two fluids are immiscible at ambient pressure. During impact one may create enough force or pressure to separate molecules, e.g. say $\mathrm{CH}_{3} \mathrm{OH}$, and insert for example $\mathrm{C}_{16} \mathrm{H}_{24}$ or $\mathrm{C}_{10} \mathrm{H}_{32}$ molecules. Actually, miscibility behav-
[18] C.H. Wang, C.Z. Lin, W.G. Hung, W.C. Huang, C.K. Law, Combust. Sci. Technol. 176 (2004) 71-93.

[19] O'Rourke, F.V. Bracco, in: Proc. Stratefied Charge Auto. Engineering Conf., Inst. Mech. Eng., 1980, ISBN 082984693.

[20] C.H. Wang, X.Q. Liu, C.K. Law, Combust. Flame 56 (1984) 175-197.

[21] A. Lee, C.K. Law, Combust. Sci. Technol. 86 (1992) 253-265.

[22] N.D. Marsh, D.L. Zhu, M.J. Wornat, Proc. Combust. Inst. 27 (1998) 1897-1905.

[23] C.K. Law, Combust. Flame 31 (1978) 285-296.

[24] I. Glassman, Combustion. Academic Press, New York, 1996, p. 327.

ior is a function of concentration of probably $\mathrm{f}(\mathrm{T}, \mathrm{P})$. Particularly at "point" contacts, pressure could be extremely high.

Reply. The adhesion upon the impact of two droplets of nominally immiscible liquids is obviously an interesting phenomenon and merits detailed study. However, since the focus of the present work is on droplet combustion and microexplosion, we have not had the opportunity to explore the mechanism and characteristics of this adhesion phenomenon. As such, we are reluctant to speculate. We thank both commenters for their insightful questions and suggestions. 